\title{
Neurally Adjusted Ventilatory Assist in Very Prematurely Born Infants with Evolving/Established Bronchopulmonary Dysplasia
}

\author{
Sandeep Shetty, MD ${ }^{1,2}$ Katie Evans, MRCPCH ${ }^{1}$ Peter Cornuaud, Bsc ${ }^{1}$ Anay Kulkarni, FRCPCH ${ }^{10}$ \\ Donovan Duffy, MRCPCH ${ }^{1,2}$ Anne Greenough, $\mathrm{MD}^{3,4,5}$
}

${ }^{1}$ Neonatal Intensive Care Centre, St George's University Hospitals NHS Foundation Trust, London, United Kingdom

2 Department of Neonatal Medicine, St George's University of London, London, United Kingdom

${ }^{3}$ Women and Children's Health, School of Life Course Sciences, Faculty of Life Sciences and Medicine, King's College London, United Kingdom

${ }^{4}$ The Asthma UK Centre in Allergic Mechanisms of Asthma, Kings College London, London, United Kingdom

${ }^{5}$ NIHR Biomedical Research Centre at Guy's and St Thomas' NHS Foundation Trust and King's College London, United Kingdom

\begin{abstract}
Address for correspondence Sandeep Shetty, MD, Neonatal Unit, St George's Hospital National Health Service Foundation Trust, London, SW17 0QT, United Kingdom

(e-mail: sandeep.shetty@stgeorges.nhs.uk).
\end{abstract}

AJP Rep 2021;11:e127-e131.

\section{Abstract \\ Keywords \\ - neurally adjusted ventilatory assist \\ - prematurity \\ - neonatal trigger ventilation \\ - length of hospital stay \\ - ventilation days}

Background During neurally adjusted ventilatory assist (NAVA)/noninvasive (NIV) NAVA, a modified nasogastric feeding tube with electrodes monitors the electrical activity of the diaphragm (Edi). The Edi waveform determines the delivered pressure from the ventilator.

Objective Our objective was to determine whether NAVA/NIV-NAVA has advantages in infants with evolving/established bronchopulmonary dysplasia (BPD).

Methods Each infant who received NAVA/NIV-NAVA and conventional invasive and NIV was matched with two historical controls. Eighteen NAVA/NIV-NAVA infants' median gestational age, 25.3 (23.6-28.1) weeks, was compared with 36 historical controls' median gestational age $25.2(23.1-29.1)$ weeks.

Results Infants on NAVA/NIV-NAVA had lower extubation failure rates (median: 0 [02] vs. $1[0-6] p=0.002$ ), shorter durations of invasive ventilation (median: 30.5, [1-90] vs. $40.5[11-199]$ days, $p=0.046$ ), and total duration of invasive and NIV to the point of discharge to the local hospital (median: 80 [57-140] vs. 103.5 [60-246] days, $p=0.026$ ). The overall length of stay (LOS) was lower in NAVA/NIVNAVA group (111.5 [78-183] vs. 140 [82-266] days, $p=0.019)$. There were no significant differences in BPD (17/18 [94\%] vs. $32 / 36$ [89\%] $p=0.511)$ or home oxygen rates $(14 / 18$ [78\%] vs. 23/36 [64\%] $p=0.305)$.

Conclusion The combination of NAVA/NIV-NAVA compared with conventional invasive and NIV modes may be advantageous for preterm infants with evolving/established BPD. received

June 25, 2021

accepted after revision

August 16, 2021
DOI https://doi.org/ 10.1055/s-0041-1739458. ISSN 2157-6998.

\footnotetext{
(C) 2021. The Author(s).

This is an open access article published by Thieme under the terms of the Creative Commons Attribution-NonDerivative-NonCommercial-License, permitting copying and reproduction so long as the original work is given appropriate credit. Contents may not be used for commercial purposes, or adapted, remixed, transformed or built upon. (https://creativecommons.org/ licenses/by-nc-nd/4.0/)

Thieme Medical Publishers, Inc., 333 Seventh Avenue, 18th Floor, New York, NY 10001, USA
} 
Despite improvements in survival rates of extremely preterm-born infants, the incidence of bronchopulmonary dysplasia (BPD) remains unchanged over the last two decades. ${ }^{1}$ As invasive ventilation is frequently necessary and indeed life-saving, numerous ventilator strategies have been developed to reduce damage to the developing lung. Synchronization of mechanical breaths with the patient's respiratory effort offers the theoretical benefit of improving oxygenation requirement with lower ventilator pressures, fewer air leaks, and increased patient comfort with less sedation requirements. ${ }^{2}$ During neurally adjusted ventilatory assist (NAVA)/ noninvasive (NIV)-NAVA, a modified nasogastric feeding tube with electrodes monitors the electrical activity of the diaphragm (Edi). ${ }^{3}$ The Edi waveform determines the magnitude of the delivered pressure from the ventilator. NAVA allows the infant to initiate support of inspiration and termination of inspiration, potentially allowing efficient ventilation at lower pressures. Infants breath in synchrony with the ventilator and therefore are more comfortable with less work of breathing. ${ }^{3}$ Furthermore, using the respiratory drive of the infant to control ventilation may help avoid hypocarbia and hypercarbia. ${ }^{4}$ NIV neurally adjusted ventilator assist (NIV-NAVA) allows coordination of the infant's respiratory efforts with initiation, termination, and degree of inspiratory pressure above positive end-expiratory pressure (PEEP) provided by the Maquet Servo N device. ${ }^{5}$ NIVNAVA has been successfully used clinically in neonates as a mode of ventilation to prevent intubation to allow early extubation and as a novel way to deliver nasal continuous positive airway pressure. ${ }^{6}$ It is not, however, known if this technique is better than other NIV techniques.

NIV-NAVA has not been assessed in infants with evolving/established BPD. The aim of our study was to assess whether NAVA followed by NIV-NAVA and other conventional modes of invasive and NIV ventilation had advantages over conventional flow triggered volume-targeted assist control ventilation (VTV), assist control ventilation (ACV), and synchronized intermittent mandatory ventilation (SIMV) invasive modes of ventilation and conventional NIV modes such as triggered bilevel positive airway pressure (BiPAP tr), bilevel positive airway pressure (BiPAP), continuous positive airway pressure (CPAP), and heated humidified high-flow nasal cannula oxygen (HHFNC).

\section{Materials and Methods}

A retrospective study was undertaken in infants with evolving or developed BPD. Premature infants born less than 32 weeks of gestational age requiring invasive ventilation support beyond the second week (14 days) of postnatal age were classified as infants who have evolving BPD, and premature infants born less than 32 weeks of gestational age requiring respiratory support at corrected 36 weeks of gestational age were deemed as infants who have established BPD. Each infant who received NAVA/NIV-NAVA and other conventional modes of invasive (VTV, ACV, or SIMV) and NIV ventilation (nasal intermittent positive pressure ventilation such as NIPPV, BiPAP, CPAP, or HHFNC) was matched with two other infants (controls) supported by conventional invasive and NIV ventilation. Matching was by gestational age, birth weight, sex, antenatal steroid exposure, and if inborn/or transferred ex utero. Infants were identified from a standardized electronic neonatal database (Badgernet). Data were obtained from the electronic documentation recording system and cross checked with the medical notes. NAVA/NIVNAVA was delivered by the SERVO-n Maquet Getinge ventilator and conventional ventilation (flow sensor triggered) by the Stephanie STEPHAN ventilator and NIV modes were BiPAP tr/BiPAP/CPAP (infant flow driver) and HHFNC (Vapotherm). Outcomes were extubation failure, duration of invasive and NIV ventilation, total length of hospital stay (LOS), BPD, and home supplementary oxygen rates. The study period was between June 2019 and November 2020. The infants were compared with a historical cohort born between June 2016 and January 2019. This project was registered with St George's University Hospitals NHS Foundation Trust (SGH) Audit department.

Infants were historically and routinely supported by the Stephanie ventilator (Stephan GMBH, Gackenbach, Germany). VTV, ACV, SIMV, and nontriggered NIV modes including BiPAP tr, BiPAP, CPAP (flow driver), and HHFNC were the standard ventilation modes for infants born prematurely. The standard initial mode of invasive ventilation was AC-VTV for most infants and if the clinical decision was made to change over to pressure mode then ACV or SIMV was considered based on the blood gases and gestational age of the infant. The standard initial mode of NIV ventilation was BiPAP tr/BiPAP or CPAP and changed to HHFNC based on circuit change dates and departmental policy. ${ }^{7}$ Infants were considered for extubation by the attending clinician if they had an improved work of breathing and/or acceptable blood gases and/or improvement in their $\mathrm{FiO}_{2}$ requirement as per unit protocol. From June 2019, Stephanie ventilator (Stephan GMBH, Gackenbach, Germany) ventilators were transitioned to Maquet Servo N ventilators which are currently the only ventilators which have NAVA ventilation modality. When on NAVA, the apnea time was set to 2 seconds and the upper pressure limit at least $5 \mathrm{~cm}$ $\mathrm{H}_{2} \mathrm{O}$ higher than the baseline settings. A 6-French 49- or $50-\mathrm{cm}$ Edi catheter was inserted via the orogastric route and correct positioning confirmed as per the instructions of the manufacturer using the Edi catheter positioning guide function on the ventilator (Maquet Servo-n User Manual Version 4.1). The guide function displays the retrocardiac electrocardiogram (ECG) and correct positioning was confirmed when the P waves and QRS complexes were visible in the uppermost leads and then decreased in size until the $P$ waves disappeared in the lowest lead. Colored highlighting of the central two leads appeared once the catheter was in the correct place. Once correct positioning was confirmed, the catheter was securely attached to the infant's face using an adhesive dressing. Before the infant was changed to NAVA mode from conventional ventilation (VTV, ACV, or SIMV), the NAVA level was adjusted so that the estimated pressure waveform on NAVA closely matched the actual pressure waveform on the baseline settings, 
Table 1 Demographic data

\begin{tabular}{|l|l|l|l|}
\hline & NAVA/NIV NAVA $(\boldsymbol{n}=18)$ & Control $(\boldsymbol{n}=\mathbf{3 6})$ & $\boldsymbol{p}$-Value \\
\hline BW $(\mathrm{g})$ & $685(580-1060)$ & $700(540-1060)$ & 0.7 \\
\hline GA (wk) & $25.3(23.6-28.1)$ & $25.2(23.1-29.1)$ & 0.9 \\
\hline Gender (male/total) & $10 / 18(56)$ & $22 / 36(61)$ & 1 \\
\hline Antenatal steroids (yes/total) & $17 / 18(94)$ & $34 / 36(94)$ & 1 \\
\hline In utero/ex utero (in utero/total) & $13 / 18(72)$ & $27 / 36(75)$ & 0.8 \\
\hline
\end{tabular}

Abbreviations: BW, birth weight; GA, gestational age; NAVA, neurally adjusted ventilatory assist; NIV, noninvasive.

Note: Data displayed as median (range) or $n(\%)$.

aiming for the peak Edi to be between 5 to $15 \mu \mathrm{V}$ as per the recommendations of the manufacturer. The baseline ventilator settings were used to determine the back-up settings to be used on NAVA in the absence of an Edi signal. Once weaned to NAVA level of 0.8 to $1.0 \mathrm{~cm} \mathrm{H}_{2} \mathrm{O} / \mathrm{mV}$ then infants were extubated onto NIV-NAVA mode on SERVO-n Maquet Getinge ventilator. The apnea time was set to 2 seconds and the upper pressure limit at least $5 \mathrm{~cm} \mathrm{H}_{2} \mathrm{O}$ higher than the baseline settings.

Extubation failure was defined as infants requiring intubation and ventilation within 72 hours of being extubated. The duration of overall ventilation and NIV ventilation included days spent at the local hospital after being discharged from the tertiary center. BPD was defined as need of oxygen at corrected 36 weeks of gestation as per National Institute of Child Health and Human Development (NICHD) definition ${ }^{8}$ and home oxygen included infants discharged on home oxygen from the tertiary center or the local hospital.

\section{Sample Size Calculation}

A sample size of 18 infants in the NAVAN/NIV-NAVA group and 36 controls allowed detection of a $20 \%$ reduction in the overall duration of invasive and NIV ventilation days in the NAVA/NIV-NAVA group controlled to the controls with $80 \%$ power and a significance level of 0.05 .

\section{Statistical Analysis}

Differences between the two groups were assessed for statistical significance using the Chi-square test or the Mann-Whitney test as appropriate using IBM SPPS statistical software, V.26 (IBM Corporation).

\section{Results}

Eighteen consecutive "NAVA" infants, median gestational age 25.3 (range: 23.6-28.1) weeks, were compared with 36 controls, median gestational age 25.2 (range: 23.1-29.1) weeks (-Table 1). Infants were commenced on NAVA/NIVNAVA at a median postnatal age of 20 (range: 2-95) days. Infants on NAVA/NIV NAVA had lower extubation failure rates (median: 0 [0-2] vs. 1 [0-6] $p=0.002$ ). Infants on NAVA/NIV NAVA group had a shorter duration of invasive ventilation (median: 30.5 [1-90] vs. 40.5 [11-199] days $p=0.046$ ) and total duration of invasive and NIV ventilation up to the point of discharge to home from the local hospital (median: 80 [57-140] vs. 103.5 [60-246] days $p=0.026$ ). There were no significant differences in BPD rates (17/18 [94\%] vs. 32/36 [89\%] $p=0.511$ ) and home oxygen rates $14 / 18$ [78\%] vs. $23 / 36$ [64\%] $p=0.305$ ) between infants on NAVA/NIV NAVA group and infants in the control group (-Table 2).

\section{Discussion}

We have demonstrated that in infants born very prematurely and with evolving or established BPD a combination of NAVA/NIV-NAVA compared with conventional invasive and NIV modes have lower extubation failure rates, shorter duration of invasive ventilation, total duration of ventilation (invasive and NIV) and length of hospital stay.

Premature extubation carries hazards including lung decruitment, compromised gas exchange, inspiratory muscle fatigue, and ultimately the need for reintubation. ${ }^{9}$ Indeed,

Table 2 Results by ventilatory mode

\begin{tabular}{|l|l|l|l|}
\hline & NAVA/NIV NAVA $(\boldsymbol{n}=18)$ & Control $(\boldsymbol{n}=36)$ & $\boldsymbol{p}$-Value \\
\hline Extubation failure rates $(n)$ & $0(0-2)$ & $1(0-6)$ & 0.002 \\
\hline Duration of invasive ventilation (d) & $30.5(2-95)$ & $40.5(11-199)$ & 0.046 \\
\hline Overall duration of invasive and NIV (d) & $80(57-140)$ & $103.5(60-246)$ & 0.026 \\
\hline Overall LOS (d) & $111.5(78-183)$ & $140(82-266)$ & 0.019 \\
\hline BPD & $17 / 18(94)$ & $32 / 36(89)$ & 0.511 \\
\hline Home oxygen & $14 / 18(78)$ & $23 / 36(64)$ & 0.305 \\
\hline
\end{tabular}

Abbreviations: BPD, bronchopulmonary dysplasia; LOS, length of stay; NAVA, neurally adjusted ventilatory assist; NIV, noninvasive.

Note: Data displayed as median (range) or $n(\%)$. 
rates of extubation failure in extremely preterm infants have been reported to be between 10 and $70 \%$, depending on the population studied and the time frame or criteria used to define failure. ${ }^{10,11}$ In a pilot study, ${ }^{12} 30$ mechanically ventilated preterm infants were randomized at the time of initial elective extubation to NIV-NAVA or NIPPV (nasal intermittent positive pressure ventilation). Rates of continuous successful extubation for $120 \mathrm{~h}$ were $92 \%$ in the NIV-NAVA group and $69 \%$ in the NIPPV group $(p=0.14)$. Infants extubated to NIV-NAVA remained extubated longer (median: 18 vs. 4 days, $p=0.02$ ) and experienced lower peak inspiratory pressures (PIP) than infants managed with NIPPV throughout the first 3 days after extubation. Our study showed similar results with significant lower extubation failure rates in the NAVA/NIV-NAVA group.

Our study showed significant reductions in the overall duration of invasive ventilation, total duration of ventilation (invasive and NIV), and length of hospital stay. A recent published study comparing 15 premature infants on NAVA/NIVNAVA born with birth weight of less than $1,500 \mathrm{~g}$ showed no statistically significant difference in total duration of respiratory support, severity of BPD, home oxygen therapies and length of hospital stay when compared with a historical cohort group. ${ }^{13}$ That study, ${ }^{13}$ in comparison to our study, had a relatively mature group of infants, median gestational age of 28.1 weeks, and birth weight $965 \mathrm{~g}$ versus 25.3 weeks and birth weight of $685 \mathrm{~g}$ in our study. The comparison cohort selected was by 1 to 1 matching to the NAVA patient compared with 2 to 1 in our study.

There were no statistically significant differences in the BPD or supplementary home oxygen rates between the NAVANIVNAVA and the control group. NAVA at the tertiary center was used in the majority of the patients with more severe lung disease; NAVA was commenced at a median postnatal age of 20 (range: 2-95) days and in infants who were difficult wean from conventional ventilation. Despite this, transition to NAVA ventilation was well tolerated in all patients. An increasing severe BPD trend toward the NAVA group was noted in one study (8/15 vs. 3/15); however, this was not statistically significant and similarly to our study they found no significant difference in the home oxygen therapy rates. ${ }^{13}$

\section{Limitations}

There are strengths and some limitations to our study. Within the study, the only significant change was the introduction of NAVA/ NIV-NAVA mode which is a modality specific to the Maquet ventilator. This is a retrospective study comparing to a historical cohort receiving conventional invasive and NIV modes of ventilation. There may be a variety of confounders that could influence the outcome of the study. Hence, the controls were matched for gestational age, birth weight, sex, antenatal steroid exposure, and if inborn/or transferred ex utero.

\section{Conclusion}

In conclusion, we have demonstrated that in infants born very prematurely and with evolving or established BPD that a combination of NAVA/NIV-NAVA compared with conventional invasive and NIV modes may be advantageous for preterm infants with evolving or established BPD.

Availability of Data and Materials

Data given in tables and as text in manuscript.

\section{Authors' Contributions}

S.S. and A.G. designed the study; S.S., K.E., and D.D. collected the data. P.C., S.S., and A.K. designed the statistical analysis and analyzed the data. All authors were involved in the preparation of the manuscript and approved the final manuscript as submitted.

\section{Funding}

None.

\section{Conflict of Interest}

A.G. has held grants from various manufacturers (Abbot Laboratories, MedImmune) and ventilator manufacturers (SLE). A.G. is currently receiving a nonconditional educational grant from SLE. A.G. has received honoraria for giving lectures and advising various manufacturers (Abbot Laboratories, MedImmune) and ventilator manufacturers (SLE).

\section{Acknowledgments}

We thank Mrs. Gibbons for secretarial assistance. We are grateful for the support from the clinical team, consultants and nurses for on-going support with introducing NAVA ventilation mode and support with the study.

\section{References}

1 Costeloe KL, Hennessy EM, Haider S, Stacey F, Marlow N, Draper ES. Short term outcomes after extreme preterm birth in England: comparison of two birth cohorts in 1995 and 2006 (the EPICure studies). BMJ 2012;345:e7976

2 Shetty S, Greenough A. Neonatal ventilation strategies and long-term respiratory outcomes. Early Hum Dev 2014;90(11): 735-739

3 Stein H, Firestone K. Application of neurally adjusted ventilatory assist in neonates. Semin Fetal Neonatal Med 2014;19(01): 60-69

4 Beck J, Reilly M, Grasselli G, et al. Patient-ventilator interaction during neurally adjusted ventilatory assist in low birth weight infants. Pediatr Res 2009;65(06):663-668

5 Firestone KS, Beck J, Stein H. Neurally adjusted ventilatory assist for noninvasive support in neonates. Clin Perinatol 2016;43(04): 707-724

6 Stein H, Beck J, Dunn M. Non-invasive ventilation with neurally adjusted ventilatory assist in newborns. Semin Fetal Neonatal Med 2016;21(03):154-161

7 Shetty S, Evans K, Kulkarni A, Greenough A. Impact of a care bundle on cost saving for noninvasive respiratory support for neonates. Adv Neonatal Care 2021 (e-pub ahead of print). Doi: 10.1097/ANC.0000000000000856

8 Jobe AH, Bancalari E. Bronchopulmonary dysplasia. Am J Respir Crit Care Med 2001;163(07):1723-1729

9 Sant'Anna GM, Keszler M. Weaning infants from mechanical ventilation. Clin Perinatol 2012;39(03):543-562

10 Hermeto F, Martins BM, Ramos JR, Bhering CA, Sant'Anna GM. Incidence and main risk factors associated with extubation failure 
in newborns with birth weight $<1,250$ grams. J Pediatr (Rio J) 2009;85(05):397-402

11 Giaccone A, Jensen E, Davis P, Schmidt B. Definitions of extubation success in very premature infants: a systematic review. Arch Dis Child Fetal Neonatal Ed 2014;99(02):F124-F127

12 Makker K, Cortez J, Jha K, et al. Comparison of extubation success using noninvasive positive pressure ventilation (NIPPV) versus noninvasive neurally adjusted ventilatory assist (NI-NAVA). J Perinatol 2020;40(08):1202-1210

13 Rong X, Liang F, Li YJ, et al. Application of neurally adjusted ventilatory assist in premature neonates less than 1,500 grams with established or evolving bronchopulmonary dysplasia. Front Pediatr 2020;8:110 